\title{
SPECTRAL REPRESENTATIONS FOR SOME UNBOUNDED NORMAL OPERATORS
}

\author{
BY \\ GEORGE MALTESE
}

Introduction. In a recent paper [17] we obtained a spectral representation for the bounded normal operator solutions of a certain functional equation whose special cases include the semigroup equation $\left(U_{\mu \nu}=U_{\mu} U_{\nu}\right)$ as well as many others. In this note we continue our study of the abstract functional equation expressed in terms of algebras of measures. We shall consider therefore a mapping $z \rightarrow U_{z}$ of a locally compact space $Z$ into the set of unbounded normal operators on a Hilbert space. Since the operators are not necessarily bounded, the conditions imposed on the mapping $z \rightarrow U_{z}$ vary somewhat from the conditions in the bounded case. The main result (Theorem 1) which we obtain here is a spectral representation analogous to the result valid in the bounded case $[17 ; 18$, Theorem 2, p. 8]. The methods which we use are suggested by the papers [12] and [13] of C. Ionescu Tulcea.

For the general theory and terminology of unbounded operators we refer the reader to the works of E. Hille and R. Phillips [10], C. Ionescu Tulcea [11], F. Riesz and B. Sz.-Nagy [21]. Spectral representations for semigroups of unbounded operators are also discussed in A. Devinatz [3], A. Devinatz and A. E. Nussbaum [4; 5], R. Getoor [7], C. Ionescu Tulcea and A. Simon [14] and A. E. Nussbaum [19]. Theorem 1 of this paper contains the main part of Theorem 1 in [14] (and henceforth certain of the results given in $[3 ; 4 ; 5 ; 7 ; 19]$ ).

The setting of the problem in algebras of measures is given in $\S \S 1,2$, and 5 . Spectral families of Radon measures and direct sums thereof are summarized in $\S \S 3$ and 4 . The main result is stated and proved in $\S 6$. In $\S 8$ we apply the main theorem to derive a corollary which in the bounded case extends a result of S. Kurepa [16].

1. Algebras of measures. We denote by $Z$ a locally compact space and by $K(Z)$ the vector space of continuous complex-valued functions $f$ defined on $Z$ and having compact support. For any compact set $A \subset Z$ let $M(Z, A)$ be the Banach space (endowed with the norm

$$
\left.\mu \rightarrow\|\mu\|=\sup \left\{|\mu(f)|: f \in K(Z),\|f\|_{\infty} \leqq 1\right\}\right)
$$

of all measures in $M(Z)$ having support contained in $A$. We shall consider $M(Z)$

Presented to the Society, January 26, 1963; received by the editors December 14, 1962. 
endowed with the inductive limit topology [see N. Bourbaki, Espaces vectoriels topologiques, Chapter I, p. 61] of the topologies of the spaces $M(Z, A)$.

Let $\mathscr{A} \subset M(Z)$ be a vector subspace which is also an algebra with respect to a law of multiplication $(v, \mu) \rightarrow v \mu$. Suppose that there exists a subspace $\mathscr{L} \subset \mathscr{A}$ which satisfies the following two conditions:

(I) If $f$ is a continuous complex-valued function defined on $Z$ and if $\int_{\mathrm{Z}} f(z) d v(z)=0$ for all $v \in \mathscr{L}$ then $f=0$.

(II) For each $\mu \in \mathscr{L}$ there is a continuous mapping $z \rightarrow \mu_{z}$ of $Z$ into $\mathscr{A}$ such that

$$
\int_{Z} f(z) d v \mu(z)=\int_{Z} d v(z) \int_{Z} f(s) d \mu_{z}(s)
$$

for every $v \in \mathscr{L}$ and every continuous complex-valued function $f$ defined on $Z$.

2. The functional equation and its characters. In the sequel we shall find spectral representations for the unbounded normal operator solutions of the (formal) functional equation

$$
a \mathscr{X}_{\mu v^{\alpha}}+b \mathscr{X}_{\mu v^{\beta}}=\mathscr{X}_{\mu} \int g(s) \mathscr{X}_{\phi(s)} d v(s) .
$$

By a character (with respect to the above functional equation) we shall mean a (nonidentically zero) continuous complex-valued function $\chi$ defined on $Z$ and satisfying the equations

$$
a \int \chi(z) d \mu v^{\alpha}(z)+b \int \chi(z) d \mu v^{\beta}(z)=\int \chi(z) d \mu(z) \int \chi(\phi(z)) g(z) d v(z)
$$

for all $\mu, v \in \mathscr{L}$. Here $a, b$ are complex-numbers and $\alpha, \beta, \phi$, are homeomorphisms of $\mathrm{Z}$ onto $\mathrm{Z}$ verifying the following conditions $\left({ }^{1}\right)$ :

(1) $\mu^{\alpha} \in \mathscr{L}, \mu^{\beta} \in \mathscr{L}$ for all $\mu \in \mathscr{L}$.

(2) $\mu^{\phi}=\mu$ for all $\mu \in \mathscr{L}$.

The conditions on the function $g$ are as follows( $\left.{ }^{1}\right)$ :

(3) $g$ is a continuous complex-valued function defined on $Z$ such that $g(z) \neq 0$ for all $z \in Z$.

(4) $[1 / g] \cdot \mu \in \mathscr{L}$ for all $\mu \in \mathscr{L}$.

By $E$ we shall denote the space of all characters endowed with the topology of uniform convergence on the compact sets of $Z$ and by $\tilde{E}$ we shall mean the StoneČech compactification of $E$.

REMARKs. Examples of algebras of measures and the objects $\{\mathscr{A}, \mathscr{L}, E\}$ defined above may be found in C. Ionescu Tulcea and A. Simon [14], and G. Maltese [18]. See also Yu. Berezanskī and S. Krein [1], the review by R. Godement, in Math. Reviews 12(1951), 188-189 and A. Povzner [20].

(1) The measure $\mu^{\alpha}$ is defined by the relations $\mu^{\alpha}(f)=\int f(\alpha(z)) d \mu(z)$ for $f \in K(Z)$. The measure $\nu=[1 / g] \cdot \mu$ is defined by $\nu(f)=\int f / g d \mu$ for $f \in K(Z)$. 
3. Spectral families. The operator representations which we shall obtain are conveniently expressed in terms of spectral families of bounded Radon measures. We shall therefore begin with a discussion of spectral families.

Let $T$ be a locally compact space, $H$ a Hilbert space and $\mathscr{L}(H, H)$ the algebra of all linear continuous mappings of $H$ into $H$. A family $\mathscr{F}=\left(\mu_{x, y}\right)_{x \in H, y \in H}$ of bounded Radon measures defined on $T$ is (hermitian) semi-spectral if

(HI) $x \rightarrow \mu_{x, y}$ is linear for all $y \in H$.

(HII) $\mu_{x, y}=\bar{\mu}_{y x}$ for all $x \in H, y \in H$.

(HIII) $\left\|\mu_{x, y}\right\| \leqq\|x\|\|y\|$ for all $x \in H, y \in H$.

If $\mathscr{F}$ is a semi-spectral family, then for every function $f$ which is bounded and $\mathscr{F}$-measurable $\left({ }^{2}\right)$ there exists $U_{f} \in \mathfrak{Q}(H, H)$ such that

$$
\left(U_{f} x \mid y\right)=\int_{T} f(t) d \mu_{x, y}(t) \quad \text { for all } x \in H, y \in H .
$$

If $B^{\infty}(T)$ denotes the Banach algebra (endowed with the sup norm) of all bounded complex-valued $\mathscr{F}$-measurable functions defined on $T$, then $f \rightarrow U_{f}$ is a linear continuous mapping of $B^{\infty}(T)$ into $\mathfrak{L}(H, H)$ (endowed with the uniform norm). A semi-spectral family $\mathscr{F}=\left(\mu_{x, y}\right)$ is called (hermitian) spectral if

(HIV) $g \cdot \mu_{x, y}=\mu_{U_{a} x, y}$ for all $g \in B^{\infty}(T), x \in H, y \in H$.

If $\mathscr{F}$ is a spectral family on $T$ and if $T(\mathscr{F})$ is the set of all $A \subset T$ such that the characteristic function $\psi_{A} \in B^{\infty}(T)$, then $T(\mathscr{F})$ is a tribe (tribu). If we define $P_{\mathscr{F}}(A)=U_{\psi_{A}}$ then $P_{\mathscr{F}}$ is a strongly countably additive spectral measure. The spectral family satisfies the equation $P_{\mathcal{F}}(T)=I$ (identity operator) if and only if $\left\|\mu_{x, x}\right\|=\|x\|^{2}$ for all $x \in H$. Detailed discussion of spectral families may be found in J. Dixmier [6], R. Godement [8] and C. Ionescu Tulcea [11; 13].

4. Direct sums of spectral families. The results which follow are essentially taken from C. Ionescu Tulcea [13]. Let $H$ be a Hilbert space and let $\boldsymbol{v}=\left(v_{x y}\right)_{x \in H}, y \in \boldsymbol{H}$ be a spectral family defined on the locally compact space $T$. For every function $f$ defined on $T$ and $v$-measurable let

$$
D(f, v)=\left\{x \in H: \int_{T}|f(t)|^{2} d v_{x, x}(t)<\infty\right\} .
$$

Then $D(f, v)$ is a linear space which is dense in $H$ and there is a unique normal operator $U_{f}$ having domain $D(f, v)$ and satisfying

$$
\left(U_{f} x \mid y\right)=\int_{T} f(t) d v_{x, y}(t) \quad \text { for all } x \in H, y \in H .
$$

Let $\left(H_{n}\right)_{n \in J}$ be a (not necessarily countable) family of orthogonal closed subspaces of $H$ such that $H=\oplus_{n \in J} H_{n}$. For each $H_{n}$ let $P_{n}$ be the projection mapping

(2) If $\mathscr{V}$ is a set of Radon measures we say that a function $f$ is $\mathscr{V}$-measurable if $f$ is $\mu$-measurable for every $\mu \in \mathscr{V}$. 
of $H$ onto $H_{n}$. For every $n \in J$ let $\mathscr{F}_{n}=\left(\mu_{x, y}^{n}\right)_{x \in H . y \in H}$ be a spectral family on $T$. Then since we have

$$
\sum_{n \in J}\left\|\mu_{P_{. . x}, P_{n} y}^{n}\right\| \leqq \sum_{n \in J}\left\|P_{n} x\right\|\left\|P_{n} y\right\| \leqq\|x\|\|y\|
$$

for all $x, y \in H$, it follows that the family $\left(\mu_{P_{n} x, P_{n} y}^{n}\right)_{n \in J}$ is absolutely summable with respect to the norm $\mu \rightarrow\|\mu\|$. Define now the measures

$$
\mu_{x, y}=\sum_{n \in J} \mu_{P_{n} x, P_{n} y}^{n}
$$

The family $\mathscr{F}=\left(\mu_{x, y}\right)_{x \in H, y \in H}$ will be called the direct sum of the families $\left(\mathscr{F}_{n}\right)_{n \in J}$. Let us now state the following proposition and refer to C. Ionescu Tulcea [13, p. 104] for its proof.

Proposition 1. (1) $\mathscr{F}$ is a spectral family; (2) $f$ is $\mathscr{F}$-measurable if and only if $f$ is $\mathscr{F}_{n}$-measurable for each $n \in J ;(3) x \in D(f, \mathscr{F})$ if and only if $P_{n} x \in D\left(f, \mathscr{F}_{n}\right)$ for each $n \in J$ and the family $\left(\int_{T}|f(t)|^{2} d \mu_{P_{n} x}^{n}, P_{n} x(t)\right)_{n \in J}$ is summable; (4) for $x \in D(f, \mathscr{F})$ and $y \in H$ we have

$$
\int_{T} f(t) d \mu_{x, y}(t)=\sum_{n \in J} \int_{T} f(t) d \mu_{P_{. . x}, P_{n} y}(t) .
$$

5. Unbounded normal operators. Let $H$ be a Hilbert space and consider the object $\{Z, \mathscr{A}, \mathscr{L}, E\}$ defined above. Let $z \rightarrow U_{z}$ be a mapping of $Z$ into the set of (not necessarily bounded) operators on $H$ which satisfies the following conditions:

$(\mathfrak{U I})\left(^{3}\right) \quad z \rightarrow\left(U_{z} x \mid y\right)$ is continuous for $x \in \Delta=\bigcap_{z \in Z} D\left(U_{z}\right)$ and $y \in H$.

$(\mathfrak{U I I})\left({ }^{4}\right) a\left(U_{\mu v^{\alpha}} x \mid y\right)+b\left(U_{\mu \nu} \beta x \mid y\right)=\left(U_{\mu} \int g(s) U_{\phi(s)} d v(s) x \mid y\right)$ for all $x, y \in \bigcap_{\mu, v \in \mathscr{L}} D\left(U_{\mu} \int g(s) U_{\phi(s)} d v(s)\right)$.

(UIII) $\left({ }^{5}\right)$ There exists $e \in Z$ such that $U_{e}=I$ (identity operator).

(UIIV) $\left\{U_{z}: z \in Z\right\}$ is a family of commuting normal (not necessarily bounded) operators.

( $\mathfrak{U V})$ There exists a countable set $Z_{0} \subset Z$ such that $\bigcap_{z \in Z} D\left(U_{z}\right)=\bigcap_{z \in Z_{0}} D\left(U_{z}\right)$. The collection of all objects $\left\{H, U_{z}\right\}$ which satisfy conditions (U्UI) through ( $\mathfrak{U V}$ ) will be denoted by $\mathfrak{N}_{2}$.

(3) $D\left(U_{z}\right)$ denotes the domain of $U_{z}$.

(4) Here $a, b, \alpha, \beta, \phi, g$ are as defined in $\S 2$. For every $\delta \in \mathscr{L}$ the operator $U_{\delta}$ is defined by the equations $\left(U_{\delta} x \mid y\right)=\int\left(U_{z} x \mid y\right) d \delta(z)$ for $x \in \Delta, y \in H$. Hence $\Delta$ is the domain of $U_{\delta}$. Similarly the operator $\int g(s) U_{\phi(s)} d \delta(s)$ is defined by $\left(\int g(s) U_{\phi(s)} d \delta(s) x \mid y\right)=\int\left(g(s) U_{\phi(s)} x \mid y\right) d \delta(s)$ for $x \in \Delta$ and $y \in H$, so that this operators also has $\Delta$ as its domain

(5) This assumption is not essential and can be avoided at the expense of more complicated arguments in the ensuing theory. For the general situation one can adapt arguments similar to those of C. Ionescu Tulcea [13, pp. 100-101]. 
REMARK. Under the assumption of condition ( $\mathfrak{U I})$ the condition $(\mathfrak{U} I I)$ is implied by the following:

$(\mathfrak{U I I})^{\prime} \quad a U_{\mu \nu} \alpha+b U_{\mu \nu} \beta \subset U_{\mu} \int g(s) U_{\phi(s)} d v(s)$.

In fact if $x \in \bigcap_{\mu, v \in \mathscr{L}} D\left(U_{\mu} \int g(s) U_{\phi(s)} d v(s)\right)$ then $x \in D\left(\int g(s) U_{\phi(s)} d v(s)\right)$ for every $v \in \mathscr{L}$ and hence $x \in \Delta$. We deduce easily that $x \in D\left(U_{\mu v^{\alpha}}\right)$ and $x \in D\left(U_{\mu \nu} \beta\right)$ and therefore by (UIII)' we have

$$
a U_{\mu v^{\alpha}} x+b U_{\mu \nu} \beta x=U_{\mu} \int g(s) U_{\phi(s)} d v(s) x
$$

and this gives (UUII) in an even stronger form.

In the course of the proof of our main theorem we shall appeal essentially to the following proposition whose proof is given in C. Ionescu Tulcea [13, p. 106].

Proposition 2. Let $\mathscr{E}$ be a family of commuting normai (not necessarily bounded) operators on a Hilbert space H. Suppose that there exists a countable subset $\mathscr{E}_{0} \subset \mathscr{E}$ such that $\bigcap_{A \in \mathscr{E}} D(A)=\bigcap_{A \in \dot{B}_{0}} D(A)$. Then there exists an orthogonal family of hermitian projections $\left\{P_{n}: n \in J\right\}$ such that (1) $\sum_{n \in J} P_{n}=I$; (2) $P_{n} H \subset D(A)$ for every $n \in J$ and $A \in \mathscr{E}$; (3) $A P_{n} \supset P_{n} A$ for every $n \in J$ and $A \in \mathscr{E}$; (4) $x \in D(A)$ if and only if the family $\left\{A P_{n} x: n \in J\right\}$ is summable. For $x \in D(A)$ we have $A x=\Sigma_{n \in J} A P_{n} x$.

6. The spectral representation. The main result which we shall establish is the following:

THEOREM 1. If $\left\{H, U_{z}\right\}$ is in the class $\mathfrak{N}_{2}$ there exists a spectral family $\mathscr{F}=\left(\mu_{x y}\right)_{x \in \boldsymbol{H}, y \in \boldsymbol{H}}$ defined on $\tilde{E}$ and concentrated on $E$ such that

$$
\left(U_{z} x \mid y\right)=\int_{\widetilde{E}} \chi(z) d \mu_{x, y}(\chi) \quad \text { for all } z \in Z, x \in D\left(U_{z}\right) \text { and } y \in H \text {. }
$$

Our method of proof will consist in showing that suitable restrictions of the operators $U_{z}$ are bounded. Then we show that these bounded restrictions belong to a class $\mathfrak{N}_{1}$ for which a spectral representation theorem is available. Finally we use the direct sum theory to obtain the representation for the members of $\mathfrak{N}_{2}$.

We shall say that an object $\left\{H, U_{s}\right\}$ is in the class $\mathfrak{N}_{1}$ if the mapping $s \rightarrow U_{s}$ of $Z$ into $\mathfrak{L}(H, H)$ satisfies the conditions:

(CI) $s \rightarrow\left(U_{s} x \mid y\right)$ is continuous for every $x, y \in H$.

(CII) $a U_{\mu v^{\alpha}}+b U_{\mu \nu} \beta=U_{\mu} \int g(s) U_{\phi(s)} d v(s)$ for all $\mu, v \in \mathscr{L}$.

(CIII) $U_{e}=I$ for some $e \in Z$.

(CIV) $\left\{U_{s}: s \in Z\right\}$ is a commuting family of bounded normal operators.

We shall make strong use of the following result (see G. Maltese $[17 ; 18$, p. 8]):

THEOREM 2 (BOUNDED CASE). Let $\left\{H, U_{s}\right\}$ be an object in the class $\mathfrak{N}_{1}$. There exists a spectral family $\mathscr{G}=\left(v_{x, y}\right)_{x \in H, y \in H}$ defined on $\tilde{E}$ and concentrated on $E$ such that 


$$
\left(U_{s} x \mid y\right)=\int_{\tilde{E}} \chi(s) d v_{x, y}(\chi) \quad \text { for all } s \in Z, x \in D\left(U_{s}\right), y \in H
$$

Proof of Theorem 1. If we let $\mathscr{E}=\left\{U_{z}: z \in Z\right\}$ then conditions (UIV) and $(\mathfrak{U V})$ imply that $\mathscr{E}$ satisfies the hypothesis of Proposition 2. Denote by $\left\{P_{n}\right\}_{n \in J}$ the orthogonal family of hermitian projections whose existence is assured by Proposition 2. For each $n \in J$, define a mapping $U_{z, n}$ by the relations

$$
U_{z, n} x=U_{z} P_{n} x
$$

for $x=P_{n} x \in H_{n}=P_{n}(H)$. The mapping $U_{z, n}$ is well defined everywhere on $H_{n}$ since if $x \in H_{n}$, then $x=P_{n} x$ so that $x \in D\left(U_{z}\right)$. But by Proposition $2, P_{n} U_{z} \subset U_{z} P_{n}$ so that for $x \in H_{n}$ we have $P_{n} U_{z} x=U_{z} P_{n} x=U_{z, n} x$ and hence $U_{z, n} x \in H_{n}$. This therefore shows that $U_{z, n}$ maps $H_{n}$ into $H_{n}$. It is now obvious that $U_{z, n}$ is closed and hence (by the Closed Graph Theorem) bounded. We proceed now to show that $\left\{U_{z . n}, H_{n}\right\} \in \mathfrak{N}_{1}$.

(i) First we shall show that the mapping $z \rightarrow\left(U_{z, n} x \mid y\right)$ is continuous for all $x, y \in H_{n}$. If $x, y \in H_{n}$ then from Proposition 2 we have that $x, y \in \Delta$. Consequently (UXI) implies that $z \rightarrow\left(U_{z} x \mid y\right)=\left(U_{z} P_{n} x \mid y\right)=\left(U_{z, n} x \mid y\right)$ is continuous and so condition (CI) is satisfied.

(ii) For every $\mu \in \mathscr{L}$ a mapping $U_{\mu, n}$ is defined by the equations

$$
\left(U_{\mu, n} x \mid y\right)=\int\left(U_{z, n} x \mid y\right) d \mu(z)
$$

for $x, y \in H_{n}$. Obviously $U_{\mu, n}$ is a bounded linear operator on $H_{n}$ for every $\mu \in \mathscr{L}$, $n \in J$. From $\left({ }^{6}\right)$ this definition it follows that $U_{\mu, n} x=U_{\mu} x$ for $x \in H_{n}$. The verification of the functional equation (CII) is now at hand. In fact for $\mu, v \in \mathscr{L}$ and $x, y \in H_{n}$, we have

$$
\begin{aligned}
\left(U_{\mu n} \int g(s) U_{\phi(, n} d v(s) x \mid y\right) & =\left(U_{\mu} \int g(s) U_{\phi(\cdot)} d v(s) x \mid y\right) \\
& =a\left(U_{\mu v^{\alpha}} x \mid y\right)+b\left(U_{\mu v} \beta x \mid y\right) \\
& =a\left(U_{\mu v^{\alpha}{ }_{n}} x \mid y\right)+b\left(U_{\mu \nu}{ }_{n} x \mid y\right) .
\end{aligned}
$$

(iii) We shall now show that for each $z \in Z$ and $n \in J$ the bounded operators $U_{z n}$ are normal. Let $x \in H_{n}$, then

$$
\left\|U_{z, n} x\right\|=\left\|U_{z} x\right\|=\left\|U_{z}^{*} x\right\|=\left\|U_{z}^{*} P_{n} x\right\|=\left\|U_{z, n}^{*} x\right\|
$$

so that the bounded operators $U_{z, n}$ are normal and (CIV) is verified. Therefore we have proved that for each $n \in J$ the objects $\left\{H_{n}, U_{z, n}\right\}$ are in the class $\mathfrak{N}_{1}$.

(6) We use here the fact that $U_{\mu} x \in H_{n}$ if $x \in H_{n}$. This can be proved as follows: let $x \in H_{n}$ and $y \in H$, then $\left(P_{n} U_{\mu} x \mid y\right)=\int\left(U_{z} x \mid y\right) d \mu(z)=\left(U_{\mu} x \mid y\right)$. Hence since $y$ is arbitrary we have $P_{n} U_{\mu} x=U_{\mu} x$. 
(iv) By the spectral representation theorem for the class $\mathfrak{N}_{1}$ (Theorem 2) there exists a spectral family $\mathscr{F}_{n}=\left(\mu_{x, y}^{n}\right)_{x \in H} \ldots y \in \boldsymbol{H}$ defined on $\tilde{E}$ and concentrated on $E$ such that for $z \in Z, x, y \in H_{n}$ we have

$$
\left(U_{z, n} x \mid y\right)=\int_{\widetilde{E}} \chi(z) d \mu_{P_{n} x P_{n} y}(\chi) .
$$

Let $\mathscr{F}$ be the direct sum of the family $\left(\mathscr{F}_{n}\right)_{n \in J}$. Then for $x \in H, z \in Z, n \in J$ we obtain

$$
\left\|U_{z} P_{n} x\right\|^{2}=\int_{\tilde{E}}|\chi(z)|^{2} d \mu_{P_{n} x, P . . x}^{n}(\chi) .
$$

If $x \in D\left(U_{z}\right)$ then by Proposition 2 the family $\left\{U_{z} P_{n} x: n \in J\right\}$ is summable. But this is an orthogonal family of vectors so that

$$
\infty>\sum_{n \in J}\left\|U_{z} P_{n} x\right\|^{2}=\sum_{n \in J} \int_{\widetilde{E}}|\chi(z)|^{2} d \mu_{P_{n} x, P_{n} x}^{n}(\chi) .
$$

Hence if $x \in D\left(U_{z}\right)$ then Proposition 1 implies that $x \in D(z(\cdot), \mathscr{F})\left({ }^{7}\right)$. Applying Propositions 1 and 2 we have for $x \in D\left(U_{z}\right)$ and $y \in H$

$$
\begin{aligned}
\left(U_{z} x \mid y\right) & =\left(\sum_{n \in J} U_{z} P_{n} x \mid y\right)=\sum_{n \in J}\left(U_{z} P_{n} x \mid y\right) \\
& =\sum_{n \in J} \int_{\widetilde{E}} \chi(z) d \mu_{P_{n} x, P_{n} y}(\chi)=\int_{\widetilde{E}} \chi(z) d \mu_{x, y}(\chi) .
\end{aligned}
$$

This completes the proof of the theorem.

7. A special case. Suppose that $Z$ is locally compact Abelian group with Haar measure $m$. Let $\mathscr{A}=\mathscr{L}$ be the vector subspace of $M(Z)$ which consists of those Radon measures $v$ having the form $v=g \cdot m$, where $g$ is an $m$-integrable function with compact support. For any measures $v, \mu \in \mathscr{L}, v=g \cdot m$ and $\mu=h \cdot m$ define $v \mu=(g * h) \cdot m$, where $*$ denotes convolution. When endowed with this multiplication $\mathscr{L}$ is an algebra and in fact may be identified with a subalgebra of the group algebra $L^{1}(Z, m)$. For every $\mu=h \cdot m \in \mathscr{L}$ and $z \in Z$ let $\mu_{z}$ denote the mapping $\left({ }^{8}\right) f \rightarrow \breve{h} * f(z)$. If we consider on $\mathscr{L}$ the topology defined by the norm $\mu \rightarrow\|\mu\|$ then the mapping $z \rightarrow \mu_{z}$ is continuous from $Z$ into $\mathscr{L}$.

Let $H$ be a Hilbert space and consider a mapping $z \rightarrow U_{z}$ of $Z$ into the set of

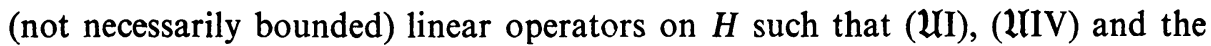
following conditions are satisfied (for all $s, t \in Z$ ):

$(\mathfrak{U I I I})^{\prime} a U_{s+\alpha(t)}+b U_{s+\beta(t)} \subset U_{s} g(t) U_{\phi(t)}$.

This implies that condition (U्UII) is also satisfied. In fact, taking

(7) $z(\cdot)$ denotes the function $\chi \rightarrow \chi(z)$.

(8) The function $\breve{f}$ is defined by the equation $\breve{f}(z)=f\left(z^{-1}\right)$ for all $z \in Z$. 


$$
x, y \in \bigcap_{\mu \in \mathscr{L}} D\left(U_{\mu} \int g(s) U_{\phi(s)} U_{\phi(s)} d v(s)\right)
$$

we have $\left({ }^{9}\right)$ that $x, y \in \Delta$ and hence

$$
\left(a U_{s+\alpha(t)} x \mid y\right)+\left(b U_{s+\beta(t)} x \mid y\right)=\left(U_{s} g(t) U_{\phi(t)} x \mid y\right) .
$$

Since the domain of a normal operator is identical with the domain of its adjoint, the right-hand term of (1) may be written as $\left(g(t) U_{\phi(t)} x \mid U_{s}^{*} y\right)$. Integrating with respect to $v \in \mathscr{L}$ we obtain

$$
\begin{aligned}
& \int\left(a U_{s+t} x \mid y\right) d v^{\alpha}(t)+\int\left(b U_{s+t} x \mid y\right) d v^{\beta}(t) \\
& =\left(\int g(t) U_{\phi(t)} d v(t) x \mid U_{s}^{*} v\left(=\left(U_{s} \int g(t) U_{\phi(t)} d v(t) x \mid y\right) .\right.\right.
\end{aligned}
$$

Finally integration with respect to $\mu \in \mathscr{L}$ yields (UIII).

8. An application. In this paragraph we consider first objects $\left\{H, U_{z}\right\} \in \mathfrak{N}_{2}$ where $Z$ is the real line, $\alpha(z)=z, \beta(z)=-z, a=b=1, g(z)=2$ and $\phi(z)=z$.

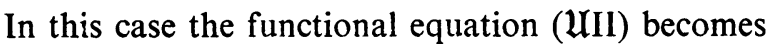

$$
\left(U_{s+t} x \mid y\right)+\left(U_{s-t} x \mid y\right)=2\left(U_{s} U_{t} x \mid y\right) \text {. }
$$

Using essentially the proof as in $[18$, p. 13 , Theorem 3$]$ we can prove the following corollary to our spectral representation theorem (for the bounded case see also S. Kurepa [16]);

Corollary. Let $Z$ be the real line and let $\left\{H, U_{z}\right\}$ be in the class $\mathfrak{R}_{2}$. There exists a spectral family $\mathscr{G}=\left(v_{x, y}\right)_{x \in H, y \in H}$ defined on the complex plane $C$ such that

$$
\left(U_{s} x \mid y\right)=\int_{C} \cos \lambda s d v_{x, y}(\lambda) \quad \text { for all } s \in Z, x \in D\left(U_{s}\right), y \in H .
$$

As an example of an object $\left\{H, U_{z}\right\}$ in the class $\mathfrak{N}_{2}$ we may take $Z$ to be the space of integers with the discrete topology and with the parameters $\alpha, \beta, \phi, g$, $a$ and $b$ defined as above. For the Hilbert space we take the $L^{2}$-space of the complex plane with respect to Lebesgue measure. For every integer $p$ define an operator

$$
U_{p}: x(\cdot) \rightarrow(\cos p \cdot) x(\cdot)
$$

for all those $x \in L^{2} \operatorname{such}$ that $(\cos p \cdot) x(\cdot) \in L^{2}$. The domain of $U_{p}$ is dense in $L^{2}$ and $U_{p}$ is an unbounded normal operator in the space $L^{2}$. This fact together with the countability and discreteness of $Z$ imply immediately that the mapping $p \rightarrow U_{p}$ satisfies all conditions for the class $\mathfrak{N}_{2}$.

ACKNOWLEDGEMENTS. This paper was supported in part by National Science Foundation Grant 19684. The author is indebted to Professor C. Ionescu Tulcea of the University of Pennsylvania for many stimulating conversations concerning the subject matter.

(9) Condition (UIII)' implies that $\Delta \subset D\left(a U_{s+\alpha(t)}+b U_{s+\beta(t)}\right) \subset D\left(U_{s} g(t) U_{\phi(t)}\right)$. 


\section{BIBLIOGRAPHY}

1. Yu. Berezanski1 and S. Krein, Hypercomplex systems with continual bases, Uspehi Mat. Nauk 12 (1957), no. 1 (73), 147-152. (Russian)

2. N. Bourbaki, Intégration, Chap. I-V (1952-1956).

3. A. Devinatz, A note on semi-groups of unbounded self-adjoint operators, Proc. Amer. Math. Soc. 5 (1954), 101-102.

4. A. Devinatz and A. E. Nussbaum, On the permutability of normal operators, Ann. of Math. (2) 65 (1957), 144-152.

5. - Real characters of certain semi-groups with applications, Duke Math. J. 28 (1961), 221-238.

6. J. Dixmier, Les algèbres d'opérateurs dans l'espace hilbertien, Gauthier-Villars, Paris, 1957.

7. R. Getoor, On semi-groups of unbounded normal operators, Proc. Amer. Math. Soc. 7 (1956), 387-391.

8. R. Godement, Sur une généralisation d'un théorème de Stone, C. R. Acad. Sci. Paris 218 (1944), 901-903.

9. __ Sur la théorie des représentations unitaires, Ann. of Math. (2) 53 (1951), 68-124.

10. E. Hille and R. Phillips, Functional analysis and semi-groups, Amer. Math. Soc. Colloq. Publ. Vol. 31, Amer. Math. Soc., Providence, R.I., 1957.

11. C. Ionescu Tulcea, Hilbert space, Editura Academici Republicii Populare Romine, 1956. (Roumanian)

12. - Spectral representations of semi-groups of normal operators, Proc. Nat. Acad. Sci. U.S.A. 44 (1958), 44-45.

13. - Spectral representations of certain semi-groups of operators, J. Math. Mech. 8 (1959), 95-110.

14. C. Ionescu Tulcea and A. Simon, Spectral representations and unbounded convolution operators, Proc. Nat. Acad. Sci. U.S.A. 45 (1959), 1765-1767.

15. — Generalized convolution algebras (unpublished manuscript).

16. S. Kurepa, A cosine functional equation in Hilbert space, Caı ad. J. Math. 12 (1960), 45-50.

17. G. Maltese, Spectral representations for some bounded normal operators (to appear).

18. - Spectral representations for solutions of certain abstract functional equations, Compositio Math. 15 (1962), 1-22.

19. A. E. Nussbaum, Integral representation of semi-groups of unbounded self-adjoint operators, Ann. of Math. (2) 69 (1959), 133-141.

20. A. Povzner, On differential equations of Sturm-Liouville type on a half-axis, Amer. Math. Soc. Transl. No. 5 (1950).

21. F. Riesz and B. Sz.-Nagy, Functional analysis, Frederick Ungar, New York, 1955.

22. B. Sz.-Nagy, Spektraldarstellung linearer Transformationen des Hilbertschen Raumes, Springer, Berlin, 1942.

Massachusetts InSTITUTE OF TEChNOLOGy, Cambridge, MassachusetTs 